\title{
Neuropsychiatric systemic lupus erythematosus is associated with imbalance in interleukin 10 promoter haplotypes
}

\author{
M J Rood, V Keijsers, M W van der Linden, T Q T Tong, S E Borggreve, C L Verweij, \\ F C Breedveld, T W J Huizinga
}

\begin{abstract}
Objective-To investigate the association of interleukin 10 (IL10) promoter polymorphisms and neuropsychiatric manifestations of systemic lupus erythematosus (SLE).

Methods-IL10 haplotypes of 11 healthy volunteers were cloned to confirm that in the Dutch population, only the three common haplotypes (-1082/-819/-592) GCC, ACC and ATA exist. The IL10 promoter polymorphisms of 92 SLE patients and 162 healthy controls were determined. The medical records of the SLE patients were screened for the presence of neuropsychiatric involvement.
\end{abstract}

Results-All cloned haplotypes were either GCC, ACC or ATA. Forty two SLE patients had suffered from neuropsychiatric manifestations (NP-SLE). In NP-SLE patients, the frequency of the ATA haplotype is $30 \%$ versus $18 \%$ in the controls and $17 \%$ in the non-NP-SLE group (odds ratios $1.9, p=0.02$, and $2.1, p=0.04$, respectively), whereas the GCC haplotype frequency is lower in the NP-SLE group compared with controls and non-NP-SLE patients $(40 \%$ versus $55 \%$ and $61 \%$, odds ratios $0.6, p=0.02$ and $0.4 p=0.006)$. The odds ratio for the presence of NP-SLE is inversely proportional to the number of GCC haplotypes per genotype when the NP-SLE group is compared with non-NPSLE patients.

Conclusions-The IL10 locus is associated with neuropsychiatric manifestations in SLE. This suggests that IL10 is implicated in the immunopathogenesis of neuropsychiatric manifestations in SLE.

(Ann Rheum Dis 1999;58:85-89)

Genetic factors play an important part in the aetiopathogenesis of systemic lupus erythematosus (SLE). This is illustrated by the $50-60 \%$ concordance rate of SLE beween monozygotic twins. ${ }^{1}$ Furthermore, several susceptibility loci have been identified, including HLA class I and II, C4A complement null alleles, tumour necrosis factor (TNF), and Fc- $\gamma$-RIIIa polymorphisms. $^{2-6}$

Genetic dissection of SLE phenotypes has been demonstrated in murine models of SLE. In NZB/W F2 mice, genome wide searches using microsatellite based maps yielded predisposing loci for SLE. Specific combinations of genes contribute additively to the risk for a given phenotype. ${ }^{7}$ Analogous to murine strains, evidence exists that in human SLE several susceptibility loci are associated with the phenotype of renal and neuropsychiatric involvement in SLE. In SLE patients with nephritis the homozygous $176 \mathrm{~V}$ genotype of Fc- $\gamma$-RIIIa was under-represented more than fourfold compared with those without nephritis. ${ }^{6}$ Several groups have studied the Fc- $\gamma$-RIIA (CD32) polymorphisms in different populations of SLE patients. ${ }^{8-12}$ Although the data are not consistent in all populations studied, the Fc- $\gamma$-RIIA alleles are probable risk factors for lupus nephritis. Neuropsychiatric SLE has been associated with HLA-DR3, HLA-DR9, and HLA-DQ2 antigens, whereas the frequency of HLA-DR4 antigens is lower than in patients without neuropsychiatric involvement. ${ }^{13}$

The IL10 locus has been implicated in susceptibility for SLE. The interest in the association of IL10 and SLE is based on the possible lupus favouring properties of IL10. IL10 is a major $\mathrm{B}$ cell growth and differentiation factor, ${ }^{14}$ and has been shown to induce immunoglobulin production in B lymphocytes of SLE patients. ${ }^{15}$ SLE patients have a higher innate production of IL10 than controls. ${ }^{16}{ }^{17}$ Innate differences in IL10 production are associated with haplotypes of chromosome 1 as defined by microsatellites. ${ }^{18}$ In two different populations (Mexican Americans and white Scots) an allelic imbalance was found for a microsatellite $1.2 \mathrm{kB} 5$ ' of the transcription initiation site of the IL10 gene, suggesting that the IL10 locus is an important susceptibility factor for SLE. ${ }^{19} 20$

In the IL10 promoter three different biallelic polymorphisms have been identified: -1082 G/A, -819 C/T and -592 C/A. Genotyping of a relatively large number of people revealed the existence of only three haplotypes, GCC, ACC, and ATA (-1082/-819/-592 respectively). ${ }^{21} 22$ IL10 promoter polymorphisms have been reported to be associated with various SLE phenotypes. A small study reported an association of the $-819 \mathrm{C}$ and $-592 \mathrm{C}$ IL10 alleles with the presence of anti-Ro antibodies in SLE. ${ }^{22}$ A recent study suggested that in southern Chinese patients the IL10 promoter haplotype $-1082 \mathrm{~A} /-819 \mathrm{~T} /-592 \mathrm{~A}$ is associated with renal involvement in SLE. ${ }^{23}$

To investigate if one of the three common IL10 promoter haplotypes is associated with the phenotype of neuropsychiatric SLE, we first cloned the IL10 promoter of 11 different people to verify that indeed only three 
Table 1 Demographic and clinical characteristics of the SLE patients

\begin{tabular}{llll}
\hline & $\begin{array}{l}N P-S L E \\
\left(n=42^{*}\right)\end{array}$ & $\begin{array}{l}n \text { no-NP-SLE } \\
\left(n=50^{\star}\right)\end{array}$ & p valuet \\
\hline Female sex (\%) & 84 & 90 & 0.9 \\
Age (y) (SD) & $39(14)$ & $39(15)$ & NA \\
SLE duration (y) (SD) & $9.3(5.6)$ & $7.0(6.1)$ & NA \\
Arthritis (\%) & 86 & 88 & 0.7 \\
Malar rash (\%) & 52 & 36 & 0.1 \\
Discoid lesions (\%) & 14 & 24 & 0.2 \\
Photosensitivity (\%) & 29 & 48 & 0.06 \\
Oral ulcers (\%) & 26 & 28 & 0.8 \\
Pericarditis (\%) & 19 & 24 & 0.6 \\
Pleuritis (\%) & 50 & 41 & 0.4 \\
Proteinuria (\%) & 24 & 32 & 0.4 \\
Leuco/erythrocyturia/cylinders (\%) & 40 & 38 & 0.8 \\
Thrombopenia (\%) & 26 & 24 & 0.8 \\
Leucopenia (\%) & 43 & 34 & 0.7 \\
ANA (\%) & 98 & 96 & 0.04 \\
Anaemia (\%) & $74(\mathrm{n}=23)$ & $44(\mathrm{n}=25)$ & 0.02 \\
Anti-ds-DNA (\%) & $86(\mathrm{n}=36)$ & $63(\mathrm{n}=49)$ & 0.4 \\
Anti-Sm antibodies (\%) & $9(\mathrm{n}=11)$ & $20(\mathrm{n}=15)$ & 0.6 \\
Anticardiolipin antibodies (\%) & $77(\mathrm{n}=31)$ & $55(\mathrm{n}=29)$ & 1.0 \\
LE test (\%) & $89(\mathrm{n}=18)$ & $89(\mathrm{n}=27)$ & \\
\hline
\end{tabular}

^Number of patients of whom presence or absence of specific feature is known, unless stated otherwise; $\nmid \chi^{2}$ testing without Bonferroni correction for multiple comparisons; NA: not applicable.

haplotypes of the IL10 promoter exist in the Dutch population. Next, 92 SLE patients were divided into a phenotype with and without neuropsychiatric manifestations. The distribution of the IL10 promoter haplotypes in these groups was studied and compared with healthy controls.

\section{Methods}

PATIENTS

Blood was collected from 92 consecutive white SLE patients of the rheumatology department of the Leiden University Medical Centre. All patients fulfilled the 1982 American College of Rheumatology criteria for SLE. ${ }^{24}$ The medical records of these patients were screened for evidence of the presence of neuropsychiatric involvement and cumulative SLE criteria. Neuropsychiatric involvement was considered to be present if formal neurological, psychiatric or neuropsychological testing by consulting physicians proved to be abnormal and no other explanation for the neuropsychiatric manifestations could be found. Forty two patients had a history of neuropsychiatric SLE (NP-SLE), 50 SLE patients had never experienced neuropsychiatric manifestations (non-NP-SLE). The control population existed of 162 healthy Dutch white volunteers.

Blood was taken from another 11 healthy controls for cloning of the IL10 promoter haplotypes.

\section{MATERIALS}

DNA was isolated from SDS-lysed and proteinase- $\mathrm{K}$ treated peripheral blood cells by phenol-chloroform extraction. Fragments of the IL10 promoter were amplified using the primer combination ATC.CTC.GAG. CCT.TCT.TTT.GCA.AGT.CTG. T ${ }^{+31}$ and GAC.GGT.ACC.CTG.ACT.ATA.GAG.TGG. $\mathrm{CAG}^{-1413}$. PCR fragments were subsequently dot blotted on Hybond $\mathrm{N}+$ membranes and hybridised according to described methods. ${ }^{25}$ Sequences of the oligonucleotides and washing temperatures were as follows: for $-1082 \mathrm{~A}$ : ACT.TCC.CCT.TCC.CAA.AGA.A, $\quad 52^{\circ} \mathrm{C}$; for $-1082 \mathrm{G}$ : TTC.TTT.GGG.AGG.GGG.
AAG, $51^{\circ} \mathrm{C}$; for $-819 \mathrm{C}$ : CAG.GTG.ATG. TAA.CAT.CTC.TGT.GC, $62^{\circ} \mathrm{C}$; for $-819 \mathrm{~T}$ : GCA.CAG.AGA.TAT.TAC.ATC.ACC.TGT, $63^{\circ} \mathrm{C}$; for $-592 \mathrm{C}$ : CCG.CCT.GTC.CTG. TAG.GAA, $50^{\circ} \mathrm{C}$; for $-592 \mathrm{~A}$ : TTC.CTA CAG.TAC.AGG.CGG.G, $52^{\circ} \mathrm{C}$.

From 11 different people whose IL10 promoter alleles had been typed before and proved to be heterozygous, the IL10 promoter was PCR amplified using the above mentioned primer combination. The PCR fragments digested with $\mathrm{KpnI}$ and XhoI, were cloned into the plasmid vector pGL3-basic (Promega Corporation, Madison, USA). After growing into XL1 bacteria, the plasmids were isolated, dot blotted, and typed for the respective polymorphisms as described above.

\section{STATISTICAL ANALYSIS}

To detect differences in the frequencies of IL10 promoter polymorphisms, odds ratios were calculated using two by two contingency tables. $\chi^{2}$ Tests were performed to test statistical significance.

\section{Results}

CHARACTERISTICS OF SLE PATIENTS AND CONTROLS

Mean age in both the non-NP-SLE and NP-SLE patients at the time of blood collection was 39 years. On average, SLE disease duration was 7.0 years for the non-NP-SLE (SD 6.1 years) and 9.3 for the NP-SLE patients (SD 5.6 years, table 1). All SLE patients were of white origin. The control group consisted of 81 female and 81 male healthy Dutch white people with a mean age of 32.3 years. Table 1 shows the cumulative SLE manifestations of the patients. Coombs positive haemolytic anaemia and anti-ds-DNA antibodies were more frequent among NP-SLE patients. After Bonferroni correction for multiple comparisons, these differences did not remain statistically significant.

The neuropsychiatric manifestations of the NP-SLE patients were the following: cerebellar ataxia $(n=4)$, cerebral haemorrhage $(n=1)$, cerebral infarction $(n=8)$, chorea $(n=1)$, cognitive deterioration $(n=8)$, coma $(n=2$, both organic brain syndrome without any abnormalities confirmed by computed tomography), cranial nerve palsy $(n=14)$, depression according to DSM IV criteria $(n=6)$, paresis $(n=13)$, psychosis $(n=9)$, seizures $(n=17)$, therapy resistant headache $(n=9)$, and transverse myelitis $(n=2)$. The patients with paresis were diagnosed to suffer from transient ischaemic episodes in two cases, peripheral neuropathy resulting from vasculitis in two cases, and inflammatory central paresis attributable to SLE in nine cases. Computed tomography in one patient with transient ischaemic deficits showed old infarctions. In two of six patients with inflammatory central paresis caused by SLE who were submitted to magnetic resonance imaging, aspecific white matter lesions were found. Thirty one patients had experienced more than one manifestation. Therapy resistant headache occurred only in combination with other manifestations. 
Table 2 Number and percentage of IL10 promoter alleles in the non-NP-SLE and NP-SLE patients, the combination of these groups, and controls

\begin{tabular}{|c|c|c|c|c|c|c|c|c|}
\hline \multirow[b]{2}{*}{ Allele } & \multicolumn{2}{|c|}{ non-NP-SLE } & \multicolumn{2}{|c|}{$N P-S L E$} & \multicolumn{2}{|c|}{ SLE } & \multicolumn{2}{|c|}{ Controls } \\
\hline & $n$ & $\%$ & $n$ & $\%$ & $n$ & $\%$ & $n$ & $\%$ \\
\hline$-1082 \mathrm{G}$ & 61 & 61 & 34 & 40 & 95 & 52 & 178 & 55 \\
\hline$-1082 \mathrm{~A}$ & 39 & 39 & 50 & 60 & 89 & 48 & 146 & 45 \\
\hline$-819 \mathrm{C} /-592 \mathrm{C}$ & 83 & 83 & 59 & 70 & 142 & 77 & 265 & 82 \\
\hline$-819 \mathrm{~T} /-592 \mathrm{~A}$ & 17 & 17 & 25 & 30 & 42 & 23 & 59 & 18 \\
\hline
\end{tabular}

n: number of alleles.

Table 3 Number and percentage of IL10 promoter haplotypes in the non-NP-SLE and NP-SLE patients, the combination of these groups, and controls

\begin{tabular}{|c|c|c|c|c|c|c|c|c|}
\hline \multirow[b]{2}{*}{ Haplotype } & \multicolumn{2}{|c|}{ non-NP-SLE } & \multicolumn{2}{|c|}{$N P-S L E$} & \multicolumn{2}{|c|}{$S L E$} & \multicolumn{2}{|c|}{ Controls } \\
\hline & $n$ & $\%$ & $n$ & $\%$ & $n$ & $\%$ & $n$ & $\%$ \\
\hline GCC & 61 & 61 & 34 & 40 & 95 & 52 & 178 & 55 \\
\hline $\mathrm{ACC}$ & 22 & 22 & 25 & 30 & 47 & 26 & 87 & 27 \\
\hline ATA & 17 & 17 & 25 & 30 & 42 & 23 & 59 & 18 \\
\hline
\end{tabular}

$\mathrm{n}$ : number of haplotypes.

Table 4 Number and percentage of IL10 promoter genotypes in the non-NP-SLE and $N P-S L E$ patients, the combination of these groups, and controls

\begin{tabular}{|c|c|c|c|c|c|c|c|c|}
\hline \multirow[b]{2}{*}{ Genotype } & \multicolumn{2}{|c|}{ non-NP-SLE } & \multicolumn{2}{|c|}{$N P-S L E$} & \multicolumn{2}{|c|}{$S L E$} & \multicolumn{2}{|c|}{ Controls } \\
\hline & $n$ & $\%$ & $n$ & $\%$ & $n$ & $\%$ & $n$ & $\%$ \\
\hline GCC/GCC & 19 & 38 & 5 & 12 & 24 & 27 & 50 & 31 \\
\hline GCC/ACC & 13 & 26 & 14 & 33 & 27 & 29 & 47 & 29 \\
\hline GCC/ATA & 10 & 20 & 10 & 24 & 20 & 22 & 31 & 19 \\
\hline ACC/ACC & 3 & 6 & 2 & 5 & 5 & 5 & 13 & 8 \\
\hline ACC/ATA & 3 & 6 & 7 & 17 & 10 & 11 & 14 & 9 \\
\hline ATA/ATA & 2 & 4 & 4 & 10 & 6 & 7 & 7 & 4 \\
\hline
\end{tabular}

$\mathrm{n}$ : number of genotypes.

VERIFICATION OF GENOTYPES

All haplotypes were either $(-1082,-819,-592)$

GCC, ACC or ATA. These data confirm in the Dutch population the previous suggestions that only three different haplotypes exist.

IL10 PROMOTER POLYMORPHISMS AND SLE

The frequencies of the IL10 promoter alleles in the SLE and non-NP-SLE patients did not differ significantly from the controls (table 2). In NP-SLE patients the $-1082 \mathrm{~A}$ and $-819 \mathrm{~T} /-$ 592A alleles were more frequent than in the control population (odds ratios 1.8, $\mathrm{p}=0.02$ and $1.9, \mathrm{p}=0.02$ respectively). When NP-SLE and non-NP-SLE patients are compared, the odds ratios increase to $2.3(\mathrm{p}=0.006)$ and 2.1 $(\mathrm{p}=0.04)$.

Table 3 represents the distribution of the IL10 promoter haplotypes. The distribution of the IL10 promoter haplotypes in SLE patients did not differ from controls. Dissecting SLE patients into the non-NP-SLE and NP-SLE groups shows a frequency of the ATA haplotype in the NP-SLE patients of $30 \%$ versus $18 \%$ in the control group (odds ratio 1.9, $\mathrm{p}=0.02$ ), whereas in the non-NP-SLE group the frequency of the ATA haplotype is similar to the control group $(17 \%$ v $18 \%$, odds ratio $0.9, p=0.8)$. Conversely, the frequency of the GCC haplotype is diminished in the NP-SLE group compared with the control group $(40 \% v$ $55 \%$, odds ratio $0.6, p=0.02)$ and is the same in the non-NP-SLE group and the controls ( $61 \%$ $v 55 \%$, odds ratio $1.3, \mathrm{p}=0.3)$. Comparing NP-SLE with non-NP-SLE patients shows that the ATA haplotype prevails at the expense of the GCC haplotype (odds ratios 2.1 and 0.4 respectively, $\mathrm{p}=0.04$ and 0.006 ).

To identify a possible dose response relation of the number of ATA or GCC haplotypes and the height of the odds ratios, the study population is divided into the six possible genotypes. Genotype ATA/ATA predominates in the NP-SLE patients $(10 \%$ versus $4 \%$ in the control and non-NP-SLE groups, odds ratios not statistically significant with these low frequencies, table 4) whereas GCC/GCC is less frequently seen among NP-SLE patients (12\% versus $31 \%$ and $38 \%$ in the control and non-NP-SLE groups respectively, odds ratios $0.3, p=0.01$ and $0.2, p=0.005$ ). The odds ratios are inversely proportional to the number of GCC haplotypes when the NP-SLE group is compared with non-NP-SLE patients (2.4, $1.6,0.2$ in case of 0,1 or 2 GCC haplotypes, $\mathrm{p}=0.09,0.3$ and 0.004 respectively). Odds ratios for the ATA haplotypes show the opposite results although not statistically significant $(0.4,1.9,2.5$ in case of 0,1 or 2 ATA haplotypes, $\mathrm{p}=0.4,0.1$ and 0.05$)$.

\section{Discussion}

In this study, the IL10 promoter haplotype ATA was found to be associated with the phenotype of SLE with neuropsychiatric manifestations, whereas the GCC haplotype was less frequent in these patients. Several considerations can be made. Thus far, the presence of the three common haplotypes was based upon results from genomic typing of the polymorphisms in a number of people and small family studies. ${ }^{2122}$ In this paper we confirm these results by actually demonstrating the physical linkage of the IL10 promoter alleles into the three putative haplotypes. Recently, in southern Chinese patients a fourth haplotype GTA has been described. ${ }^{23}$ In this study the GTA haplotype could not be identified. This might be because of the fact that we deliberately restricted our study population to white patients and controls to omit racial differences as a cause for imbalance in haplotypes. At this moment no data are available to explain the existence of a limited number of haplotypes. It may be related to the function of IL10 during evolution or the tertiary structure of the IL10 promoter.

As neuropsychiatric SLE is characterised by a vast array of possible manifestations ranging from relatively mild to severe, the definition of NP-SLE is difficult. In this study, the definition of the phenotype of neuropsychiatric SLE was rather strict. In the NP-SLE group, only patients with objective manifestations have been included. Therefore, some misclassification of mild subjective neuropsychiatric manifestations to the non-NP-SLE group may have occurred. Another concern is that neuropsychiatric manifestations may be caused by different pathogenetic mechanisms with each a different immunogenetic background. In general, the different pathogenetic mechanisms of neuropsychiatric manifestations are considered to be antiphospholipid related in case of thrombotic events and immune mediated in case of diffuse manifestations. ${ }^{26}$ However, no 
evidence has been presented that confirms the supposition that the immunogenetic background of thrombotic neuropsychiatric manifestations differs from immune mediated symptoms. Nevertheless, the data were also analysed after exclusion of the nine patients suffering from manifestations with a possible thrombotic origin. The frequencies of the GCC, ACC, and ATA haplotypes in the remaining NP-SLE patients were $44 \%, 29 \%$, and $27 \%$ respectively, resulting in an odds ratio of 0.5 for the GCC haplotype in NP-SLE patients compared with non-NP-SLE patients $(p=0.03)$. The odds ratio of the ATA haplotype was 1.8 , but did not reach statistical significance because of small number of patients. These data suggest that the immunogenetic background of both patients with thrombotic as well as immune mediated pathogenesis is similar.

It has been suggested that in southern Chinese patients renal involvement in SLE patients is associated with the ATA haplotype. ${ }^{23}$ In our white population however, the three haplotypes were equally distributed among patients with and without renal disease $(54 \%$ GCC, $25 \%$ ACC, and $21 \%$ ATA versus $50 \%$ GCC, $26 \%$ ACC, and $24 \%$ ATA in patients with and without renal disease respectively). Demographic parameters such as age at onset of disease and disease duration in our and the aforementioned study are comparable. The definition of renal disease is essentially the same in both studies and thus cannot be responsible for the discrepancy in haplotype frequencies. In this study, renal disease is considered to be present if a biopsy confirmed World Health Organisation classifying diagnosis could be established (18 patients) or if patients had persistent leucocyturia, erythrocyturia, erythrocyte, or haemoglobin casts or proteinuria $>0.5 \mathrm{~g} /$ day (42 cases), whereas in the former study renal involvement is defined as the presence of either biopsy confirmed nephritis or proteinuria. Remarkably, in the southern Chinese population a trend of an increased frequency of the ATA haplotype in patients with NP-SLE is present (odds ratio $2.2, \mathrm{p}=0.08$ ), similar to our data.

The finding that an imbalance in the IL10 promoter haplotypes exists in patients with neuropsychiatric manifestations of SLE can be explained in two ways. Firstly, the imbalance may result from linkage to yet another gene at a different locus. Secondly, the imbalance is linked to a different production of IL10 leading to NP-SLE. SLE patients are reported to have a higher innate production of IL10 than controls. ${ }^{27}{ }^{28}$ We have previously demonstrated that in RA patients the $-1082 \mathrm{~A}$ allele is associated with a higher innate IL10 production than in controls. ${ }^{29}$ The current study shows an excess of the $-1082 \mathrm{~A}$ allele in the NP-SLE patients. This may indicate that in NP-SLE, the innate production of IL10 is at a higher level than in non-NP-SLE and controls. An experimental rat model has been constructed that shows that immune complex disease affects neuropsychological functioning. ${ }^{30}$ The pathogenetic mechanism in human NP-SLE might be similar to this rat model. IL10 may be at least partly responsible for the production of immune complexes in patients with NP-SLE.

In this study the IL10 locus is found to be associated with neuropsychiatric involvement in SLE. This suggests that IL10 is involved in the immunopathogenesis of this phenotype. Future studies are necessary to investigate the association of IL10 production and NP-SLE.

1 Block SR, Winfield JB, Lockshin MD, D'Angelo WA, Christian CL. Studies of twins with systemic lupus erythematosus. Am J Med 1975;59:533-52.

2 Arnett FC. The genetic basis of lupus erythematosus. In: Wallace DJ, Hahn BH, eds. Dubois'lupus erythematosus. 4th ed. Philadelphia: Lea and Febiger, 1993:13-36.

3 Truedsson L, Sturfelt G, Johansen P, Nived O, Thuresson B. Sharing of MHC haplotypes among patients with systemic lupus erythematosus from unrelated Caucasian multicase families: disease association with the extended haplotype [HLA-B8, SC01, DR17]. J Rheumatol 1995;22:1852-61.

4 Sturfelt G, Hellmer G, Truedsson L. TNF microsatellites in systemic lupus erythematosus-a high frequency of the
TNFabc 2-3-1 haplotype in multicase SLE families. Lupus 1996;5:618-22.

5 Sullivan KE, Wooten C, Schmeckpeper BJ, Goldman D, Petri M. A promoter polymorphism of tumor necrosis factor alpha associated with systemic lupus erythematosus in african-americans. Arthritis Rheum 1997;40:2207-11.

6 Wu J, Edberg J, Recheda PB, Bansai V, guyre PM, Coleman $\mathrm{K}$, et al. A novel polymorphism of Fc-gammaRIIIa (CD16) alters receptor function and predisposes to autoimmune disease. J Clin Invest 1997;100:1059-70.

7 Theofilopoulos AN. Genetics of systemic autoimmunity. J Autoimmun 1996;9:207-10.

8 Duits AJ, Bootsma H, Derksen RHWM, Spronk PE, Kater L, Kallenberg CGM, et al. Skewed distribution of IgG Rc receptor IIa (CD32) polymorphism is associated with renal disease in systemic lupus erythematosus patients. Arthritis Rheum 1995;39:1832-6.

9 Botto M, Theodoridis E, Thompson EM, Beynon HLC, Briggs D, Isenberg DA, et al. FcgammaRIIa polymorphism in systemic lupus erythematosus (SLE): no association in systemic lupus erythematosus (SLE): no associa

10 Smyth LJC, Snowden N, Carthy D, Papasteriades C, Hajeer A, Ollier WER. FcgammaRIIa polymorphism in systemic lupus erythematosus. Ann Rheum Dis 1997;56:744-6.

11 Salmon JE, Millard S, Schachter LA, Arnett FC, Ginzler EM, Gourley MF, et al. FcgammaRIIA alleles are heritable risk factors for lupus nephritis in African Americans. J Clin Invest 1996;97:1348-54

12 Song YW, Han C, Kang S, Baek H, Lee E, Shin C, et al. Abnormal distribution of Fc-gamma receptor type IIa polymorphisms in Korean patients with systemic lupus erythematosus. Arthritis Rheum 1998;41:421-6.

13 Silva LM, Donadi EA. Is immunogenetic susceptibility to neuropsychiatric systemic lupus erythematosus (SLE) different from non-neuropsychiatric SLE? Ann Rheum Dis 1996;55:544-7.

14 Rousset F, Garcia E, Defrance T, Péronne C, Vezzio N, Hsu $\mathrm{D}$, et al. Interleukin 10 is a potent growth and differentiation factor for activated human B lymphocytes. Proc Natl tion factor for activated human B

15 Llorente L, Zou W, Levy Y, Richaud-Patin Y, Wijdenes J, Alcocer-Varela J, et al. Role of Interleukin 10 in the B lymphocyte hyperactivity and autoantibody production of human systemic lupus erythematosus. J Exp Med 1995; 181:839-44

16 Llorente L, Richaud-Patin Y, Fior R, Alcocer-Varela J, Wijdenes J, Morel-Fourrier B, et al. In vivo production of interleukin-10 by non- $T$ cells in rheumatoid arthritis, Sjogren's syndrome, and systemic lupus erythematosus. Arthritis Rheum 1994;37:1647-55.

17 Hagiwara E, Gourley MF, Lee S, Klinman DM. Disease severity in patients with systemic lupus erythematosus correlates with an increased ratio of interleukin-10: interferon gamma secreting cells in the peripheral blood. Arthritis gamma secreting cells in

18 Eskdale J, Gallagher G, Verweij CL, Keijsers V, Westendorp RGJ, Huizinga TWJ. IL-10 secretion, in relation to the haplotypic structure of the human IL-10 locus. Proc Natl Acad Sci USA 1998;95:9465-70.

19 Mehrian R, Quismorio FP, Jr., Strassman G, Stimmler MM, Horwitz DA, Kitridou RC, et al. Synergistic effect between IL-10 and bcl-2 genotypes in determining sysceptibility to systemic lupus erythematosus. Arthritis Rheum 1998;41: 596-602.

20 Eskdale J, Wordsworth P, Bowman S, Field M, Gallagher G. Association between polymorphisms at the human IL-10 ocus and systemic lupus erythematosus. Tissue Antigens 1997;49:635-9.

21 Turner DM, Williams DM, Sankaran D, Lazarus M, Sinnott PJ, Hutchinson IV. An investigation of polymorphisms in the interleukin-10 gene promoter. Eur J Immunogen 1997;24:1-8.

22 Lazarus M, Hajeer AH, Turner D, Sinnott P, Worthington J, Ollier WER, et al. Genetic variation in the interleukin 10 gene promoter and systemic lupus erythematosus. J Rheumatol 1997;24:2314-17. 
23 Mok CC, Lanchbury JS, Wai Chan D, Sing Lau C. Interleukin-10 promoter polymorphisms in southern Chinese patients with systemic lupus erythematosus. Arthritis Rheum 1998;41:1090-5.

24 Tan EM, Cohen AS, Fries JF, Masi AT, McShane DJ, Rothfield NF, et al. The 1982 revised criteria for the classification of systemic lupus erythematosus. Arthritis Rheum 1982;25:1271-7.

25 Verduyn W, Doxiadis IIN, Anholts J, Drabbels JJM, Naipal A, D'Amaro J, et al. Biotinylated DRB sequence-specific oligonucleotides. Comparison to serologic HLA-DR typing of organ donors in Eurotransplant. Hum Immunol 1993;37:59-67.

26 Hama N, Boumpas DT. Cerebral lupus erythematosus. Diagnosis and rational drug treatment. CNS Drugs 1995;3:416-26.

27 Van der Linden MW, Westendorp RGJ, Sturk A, Stoeken DJ, Vandenbroucke JP, Breedveld FC, et al. Innate high
IL-10 production is associated with susceptibility for systemic lupus erythematosus (SLE). [Abstract]. Arthritis Rheum 1997;40:S307.

28 Llorente L, Richaud-Patin Y, Couderc J, Alarcon-Segovia $\mathrm{D}$, Ruiz-Soto R, Alcocer-Castillejos N, et al. Dysregulation of interleukin-10 production in relatives of patients with systemic lupus erythematosus. Arthritis Rheum 1997;40: 1429-35.

29 Keijsers V, Verweij CL, Hazes JMW, Westendorp RGJ, Breedveld FC, Huizinga TWJ. Innate differences in IL-10
production are present at the level of transcription and production are present at the level of transcription and
associated with haplotypes: Association of IL-10 haplotypes and rheumatoid arthritis. Clin Exp Rheumatol 1998; 16:200.

30 Hoffman SA, Shucard DW, Harbeck RJ. The immune system can affect learning: chronic immune complex disease in a rat model. J Neuroimmunol 1998;86:163-80.

\section{Annals of the Rheumatic Diseases - http://www.annrheumdis.com}

Visitors to the world wide web can now access Annals of the Rheumatic Diseases either through the BMJ Publishing Group's home page (http://www.bmjpg.com) or directly by using its individual URL (http:// www.annrheumdis.com). There they will find the following:

- Current contents list for the journal

- Contents lists of previous issues

- Members of the editorial board

- Subscribers' information

- Instructions for authors

- Details of reprint services

A hotlink gives access to:

- BMJ Publishing Group home page

- British Medical Association web site

- Online books catalogue

- BMJ Publishing Group books

The web site is at a preliminary stage and there are plans to develop it into a more sophisticated site. Suggestions from visitors about features they would like to see are welcomed. They can be left via the opening page of the BMJ Publishing Group site or, alternatively, via the journal page, through "about this site". 